\title{
Computing Optimal Trajectories for Medical Treatmen t Planning and Optimization
}

\author{
Ovidiu Daescu ${ }^{\star}$ and Ashish Bhatia \\ Department of Computer Science, \\ University of Texas at Dallas, \\ Richardson, TX 75083, USA, \\ \{daescu, abhatia\}outdallas.edu
}

\begin{abstract}
In this paper we discuss approximation algorithms for the 2dimensional weighted regions optimal penetration problem and propose a heuristic for speeding up the computation. The problem asks to find a ray (direction, trajectory) to access a target region in a weighted subdivision, such that some weighted distance function over the regions intersected by the ray is minimized.
\end{abstract}

\section{Introduction}

Recent developments in medical imaging ha ve made possible to provide precise information on the anatomy of a 2-dimensional (2-D, planar) or 3-dimensional (3-D, spatial, volumetric) region of the human body and on the anatomical localization and extent of "target" subregions, such as tumors, contained within that region. For example, computed tomograph y and magnetic resonance imaging can be used to provide 2-D and $3-\mathrm{D}$ data, and powerful image processing techniques can be employed to process the data for medical treatment planning (e.g., surgical planning, minimally invasive surgical methods). In this paper we assume that the planar or volumetric region has been preprocessed and partitioned into subregions, each of which has been associated a weight (based on some weighting criterion), and that one subregion has been identified as a target. The goal is to find a ray (direction, trajectory) to access the target such that some weighted distance function over the regions intersected by it is minimized.

More formally, we consider the weighted regions optimal penetration problem, introduced in [7]. The input is a subdivision $R$ in the 2-D space, composed of $r$ weighted regions $R_{i}, i=1,2, \ldots, r$, with a total of $n$ vertices. The problem is to find a ray $L$ that originates from outside $R$ and intersects a specified target region $T \in\left\{R_{1}, R_{2}, \ldots, R_{r}\right\}$, such that the weighted sum $S(L)=\sum_{L \cap R_{i} \neq \phi} w_{i} * f_{i}(L)$ is minimized, where $f_{i}(L)$ is a function associated with the pair $\left(R_{i}, L\right)$ and $w_{i}$ is a positive integer weight associated with $R_{i}$. We will call $L$ a penetrating ray with respect to the target, or penetration for short. The regions $R_{i}, i=1,2, \ldots, r$, are

\footnotetext{
* The research of this author is supported in part by the National Science Foundation under Grant EIA-0130847 and by founds from the Clark Foundation Research Initiation Grants Program.
} 


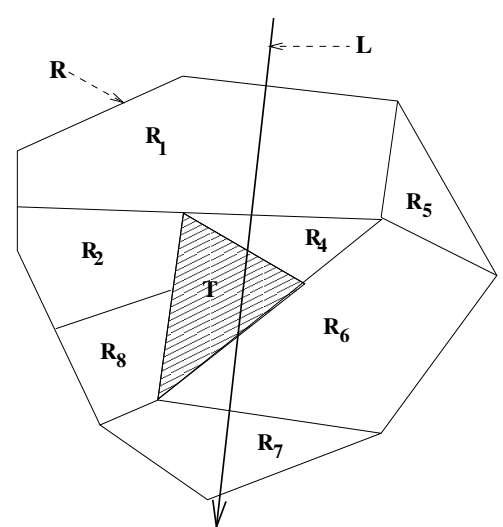

(a)

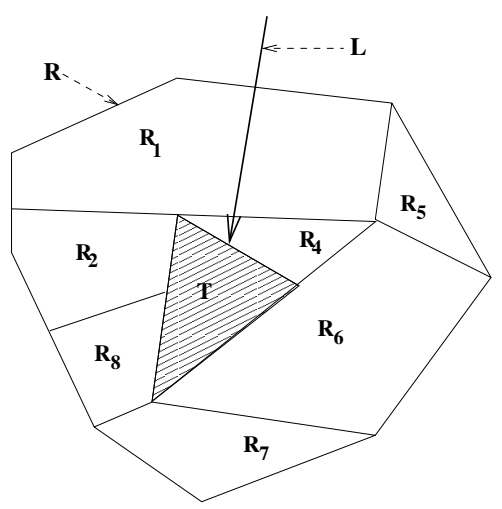

(b)

Fig. 1. The penetration $L$ : (a) goes through the target; (b) stops at the target

all convex polygons in the 2-D space, and the weights of $T$ and the complement $\bar{R}$ of $R$ are zero $\left(\bar{R}\right.$ is the free space outside $R$ ). Let $R_{L}$ denote the set of all regions of $R$ intersected by a penetration $L$ and let $d_{i}$ denote the Euclidean, $L_{2}$ length of $L$ within $R_{i} \in R_{L}$. Two versions of this problem have been introduced in [7]. In the first version (P1), $f_{i}(L)=d_{i}$ for all $R_{i} \in R_{L}$. In the second version (P2), the penetration stops when it hits the target. Thus, $f_{i}(L)$ is either $d_{i}$ or zero, depending on whether $R_{i}$ is passed by $L$ before or after $L$ intersects the target region $T$ (see Figure 1 for an example). Since the solutions to P1 and P2 are similar, we will only discuss the second version of the problem.

The penetration problem arises in several medical areas, such as radiation therapy, stereotactic brain surgery, minimally invasive computer assisted surgery and telerobotics applications in surgery $[6,3,10,13,7,14,11,2,4,5,9,12]$. For example, in radiation therapy $\mathrm{P} 1$ is related to the use of a radiation ray (beam, pencil) directed at the target from an external source (teletherapy) while P2 can be used to model the process of delivering radiation by direct implantation of a radiation source into the target (branchytherapy). In practice, finding optimal directions and trajectories is one of the most difficult problems of medical treatment optimization. While there are quite a few 2-D and 3-D results on treatment optimization with respect to other parameters, significant progress on optimizing directions has been made only recently $[7,8]$.

In a companion paper, we have proved that the optimal penetration goes through a vertex of the subdivision in the 2 - $\mathrm{D}$ case and that it is defined by a two dimensional set of lines in the 3 -D case. In this paper, we make use of those results to design approximation algorithms for the 2-dimensional weighted regions optimal penetration problem. We also propose a heuristic for speeding up the computation of the approximation algorithms. 


\section{Previous 2-D Algorithms}

A continuous ray space modeling for the 2 -D optimal penetration problem have been presented in [7], where it has been proved that the problem can be reduced to solving $O\left(n^{2}\right) 2-\mathrm{D}$ global optimization problems of the form

$$
\min _{(x, y) \in D} f(x, y)=\sqrt{1+x^{2}} \sum_{i=1}^{k} \frac{a_{i} y+b_{i}}{x+c_{i}}
$$

where $D$ is a convex $2-\mathrm{D}$ domain (a cell in an arrangement of lines), $a_{i}, b_{i}$ and $c_{i}$ are constants, the variables $x$ and $y$ are the defining parameters (slope and intercept) for the penetration $L$ and $k$ is $O(n)$ in the worst case.

Since the optimal penetration has been proved to go through a vertex of the subdivision $R$ (i.e., the optimal solution lies on the boundary of some feasible domain $D)$, the $O\left(n^{2}\right)$ 2-D global optimization problems can be reduced to solving $O\left(n^{2}\right) 1-\mathrm{D}$ global optimization problems of the form

$$
\min _{x \in D_{x}} f_{x}(x)=\sqrt{1+x^{2}}\left(d_{0}+\sum_{i=1}^{k} \frac{d_{i}}{x+c_{i}}\right)
$$

where $d_{0}, c_{i}$ and $d_{i}, i=1,2, \ldots, k$, are constants, $D_{x}$ is an interval on the $\mathrm{X}$-axis defined by some bounding edge of $D$ and $k$ is $O(n)$ in the worst case.

When using general purpose global or local optimization software as in [7], the reduction above could result in orders of magnitude speed-up for solving the 2-D penetration problem. However, for computer based medical planning systems that require fast planning algorithms (e.g., on-line surgical planning) such speedup may not be sufficient. In the next two sections, we use the knowledge that the optimal solution goes through a vertex of the subdivision and present simple and fast algorithms for finding an approximate solution.

\section{Approximation Algorithms}

In this section we present two approximation algorithms for the 2-D optimal penetration problem. Our algorithms are based on simple, yet efficient, edge and angle subdivision methods and can be refined to attend a user specified precision at an expense in computing time.

As mentioned in the previous section, a solution for the optimal penetration problem can be found by solving a number of global optimization problems. In [7, 8 ] it has been proved that the feasible domains for the 2-D global optimization problems can be generated using line (or line segment) arrangement traversal (construction) algorithms. Each 2-D feasible domain corresponds to a cell in the arrangement.

Lemma 1. The 1-D global optimization problems can be generated in a total of $O(n \log n+k+k m)$ time, where $m$ is the maximum number of terms in any of the $1-D$ objective functions and $k$ is the complexity of the arrangement. 
Proof. The boundary of each feasible domain is constructed and maintained by the arrangement traversal algorithm and thus the $O(k) 1$-D feasible domains can be obtained in $O(k)$ time by traversing the corresponding arrangement structures (usually doubly-connected edge lists). For each boundary edge $e$, the objective function can be updated in linear time in the number of fractional terms in the body of the function. Thus, if $m$ is the maximum number of terms in any of the 1-D functions, each update requires at most $\mathrm{O}(\mathrm{m})$ time.

Alternatively, the following simple and practical algorithm can be used:

1. For each vertex $v \in R$, compute the two tangent lines to the target region $T$. Let $W(v, T)$ be the double wedge defined by $v, T$, and the tangents. This computation takes $O(n \log n)$ time.

2. For each $W(v, T)$, find the set of vertices $V W(v, T)$ of $R$ that are in $W(v, T)$, which takes $O\left(n^{2}\right)$ time overall (a range searching data structure adapted from triangle range searching can be used for better theoretical bounds [1]).

3. For each vertex $v \in R$, sort the set $V W(v, T)$ around $v$ and compute the intersection of the lines defined by $v$ and $V W(v, T)$ with the boundary of $T$. This takes a total of $O(n l \log l)$ time, where $l$ is the maximum size of any set $V W(v, T)$. Observe that this set of lines includes those defined by $v$ and the vertices of $T$. Thus, we have obtained a set of $O(l)$ double wedges at $v$.

4. For each vertex $v \in R$, compute the objective function associated with the leftmost double wedge at $v$, then traverse the double wedges at $v$ while updating the objective function. The overall computation in this step can be done in $O(\mathrm{nlm})$ time, where $m$ is the maximum number of terms in any of the $O(n l)$ objective functions.

Lemma 2. The algorithm above constructs the global optimization problems for finding an optimal penetration in $O\left(n^{2}+n l m+n l \log l\right)$ time, using $O(n l m)$ space.

Proof. Follows from the algorithm description.

Some of the advantages of this algorithm are that it avoids using duality transforms and (line segment) arrangement computation for generating the optimization problems, which may lead to more robust implementations, and it can be easily adapted to generate only a subset of the optimization problems (e.g., only a subset of vertices of $R$ may be considered; that subset can be specified by the user or it can be selected based on some vertex weighting criteria).

Both algorithms above have the following two features besides simplicity. First, they allow for easy, scalable, parallel computation of the optimization problems (e.g., they can be adapted for a coarse-grain parallel model of computation as in [8]). Second, as we will show bellow for the second algorithm, they can be easily modified to produce incrementally better approximations of the optimal solution.

Let $W(v, e)$ be one of the double wedges at $v$, where $e$ is the line segment on the boundary of $T$ defined by the double wedge (see Figure 2). Let $\epsilon$ be an 


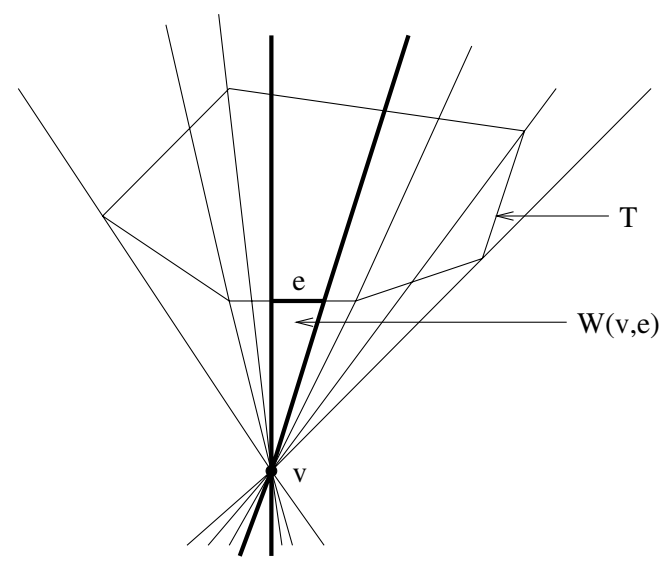

Fig. 2. The wedges at $v$ and $W(v, e)$.

input parameter of the problem and let $\lambda$ be the maximum length of any of the segments on $T$ defined by double wedges at the vertices of $R$. Let $|e|$ be the Euclidean length of $e$. We propose two strategies for refining the quality of an optimal solution. The first one is a basic subdivision method for $e$ : compute $\frac{|e|}{\epsilon} \leq \frac{\lambda}{\epsilon}$ evenly spaced points on $e$ and the corresponding double wedges. Let $\delta=\frac{\lambda}{\epsilon}$. Thus, we obtain $O(\delta)$ double wedges and need to compute $O(\delta)$ values of the penetration at evenly spaced points (on the line segment $e$ ). Note that for the 1-D optimization problem associated with $e$ we use the slope of a line through $v$ as a parameter, and thus the corresponding feasible points are not evenly spaced, in general. The second strategy we propose is a discretization of the continuum of orientations at $v$ that fall in the range of $W(v, e)$, resulting in $\delta=O\left(\frac{1}{\epsilon}\right)$ evenly spaced directions at $v$, defining $O(\delta)$ double wedges. As before, this requires computing $O(\delta)$ values of the penetration at evenly spaced points (on the feasible domain).

Lemma 3. Using the edge or angle subdivision methods, an approximate solution can be computed in $O\left(n l(m+\delta) \log ^{2} m+n l \log l+n^{2}\right)$ time.

Proof. The last two terms in the time expression follow from steps 2 and 3 of the algorithm above. A double wedge computed by the algorithm is partitioned into $O(\delta)$ double wedges, each corresponding to the same objective function. Observe that an objective function is the product of two terms and the time to compute its value at a given point is dominated by the computation of the second one (see expression (2) in the previous section). That term is a sum of $O(m)$ 1-D linear fractional functions and can be expressed as the quotient of two polynomials of degree at most $m$. Thus, it can be evaluated at $\delta$ points in a total of $O\left((m+\delta) \log ^{2} m\right)$ time, using divide and conquer and Fast Fourier Transform (FFT). 


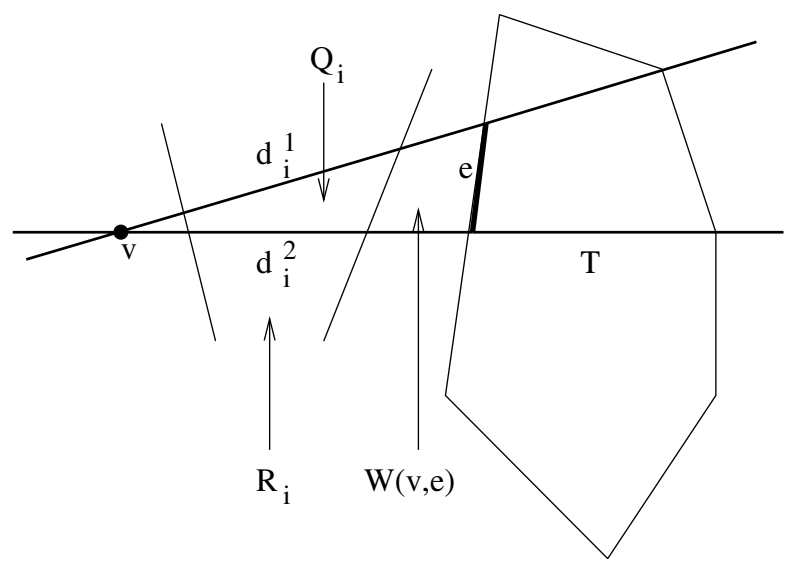

Fig. 3. A quadrilateral $Q_{i}$ at $v$ and its sides $d_{i}^{1}$ and $d_{i}^{2}$.

\section{Making Good Choices}

In this section we introduce a heuristic to help eliminate some of the optimization problems or reduce the number of approximate values computed by the approximation algorithms described earlier. The heuristic is used to guide the computation for approaching the optimal solution and can be easily incorporated into a recursive scheme for improving the quality of the approximation. More details are given bellow.

Before describing the method, we bound the quality of the approximation over a double wedge. Consider an optimization problem associated with some double wedge $W(v, e)$. Without loss of generality, let $R_{L}=\left\{R_{1}, R_{2}, \ldots, R_{j}\right\}$ be the set of regions of $R$ intersected by any ray $L \in W(v, e)$. For a quadrilateral $Q_{i}=W(v, e) \cap R_{i}$, where $R_{i} \in\left\{R_{1}, R_{2}, \ldots, R_{j}\right\}$, let $d_{i}^{1}$ and $d_{i}^{2}$ be the two sides of $Q_{i}$ that are on on the supporting lines of $W(v, e)$ (see Figure 3 ). Let $d_{i}^{\text {min }}$ be the minimum length of any line segment $s$ with endpoints on the other two sides of $Q_{i}$ such that the supporting line of $s$ goes through $v$. Similarly, let $d_{i}^{\text {max }}$ be the maximum length. Then, we have the following Lemma.

Lemma 4. $\sum_{i=1}^{j} w_{i} d_{i}^{\text {min }} \leq L_{o p t} \leq \sum_{i=1}^{j} w_{i} d_{i}^{m a x}$, where $L_{\text {opt }}$ is the value of an optimal penetration in double wedge $W(v, e)$.

Let $L_{v}^{\min }=\sum_{i=1}^{j} w_{i} d_{i}^{\text {min }}$ and $L_{v}^{\text {max }}=\sum_{i=1}^{j} w_{i} d_{i}^{\text {max }}$. We refer to $L_{v}^{\text {min }}$ and $L_{v}^{\max }$ as the lower bound and upper bound at $v$, respectively. The heuristic we propose maintains the minimum $L^{\text {min }}$ of the upper bounds over all optimization problems generated so far. A new optimization problem at some vertex $v$ is accepted if $L_{v}^{\min } \leq L^{\min }$ and rejected otherwise.

Lemma 5. The heuristic above rejects only optimization problems that cannot lead to the overall optimal solution. 
We say that an optimization problem is active if its lower bound is no larger than $L^{\text {min }}$. Optimization problems that have been accepted are maintained in a priority queue based on the value of the lower bound. Note that some of the accepted problems that are in the queue may become inactive as the computation progresses. Such problems can be eliminated from the queue by inspecting it each time the number of queued problems exceeds some predefined bound. Finally, the algorithm terminates when an optimization problem for some $W(v, e)$ is selected from the queue, such that $L^{\text {min }}-L_{v}^{\text {min }}$ is smaller than some user specified approximation error.

Currently, we are in the process of implementing our approximation algorithms and heuristic. Simulation results will be presented in the full paper.

\section{References}

1. P.K. Agarwal and J. Erickson, "Space-time tradeoffs for emptiness queries," Advances in Discrete and Comp. Geometry, (Bernard Chazelle, Jacob E. Goodman, and Richard Pollack, editors), Contemporary Mathematics, Vol. 223, pp. 1-56, 1999.

2. A.L. Boyer, T.R. Bortfeld, L. Kahler, and T.J. Waldron, "MLC modulation of xray beams in discrete steps," Proc. of the 11th Conf. on the Use of Computers in Radiation Therapy, pp. 178-179, 1994.

3. A. Brahme, "Optimization of radiation therapy" Int. J. Radiat. Oncol. Biol. Phys., Vol. 28, pp. 785-787, 1994.

4. R.D. Bucholz, "Introduction to the Journal of Image Guided Surgery" Journal of Image Guided Surgery, Vol. 1, pp. 1-11, 1995.

5. C.W. Burckhardt, P. Flury and D. Glauser, "Stereotactic Brain Surgery," IEEE Engineering in Medicine and Biology, Vol. 14, pp. 314-317, 1995.

6. Y. Censor, M.D. Altschuler and W.D. Powlis, "A computational solution of the inverse problem in radiation-therapy treatment planning," Applied Math. and Computation, Vol. 25, pp. 57-87, 1988.

7. D.Z. Chen, O. Daescu, X. Hu, X. Wu and J. Xu, "Determining an optimal penetration among weighted regions in two and three dimensions," Journal of Combinatorial Optimization, Spec. Issue on Optimization Problems in Medical Applications, Vol. 5, No. 1, pp. 59-79, 2001.

8. O. Daescu, "Parallel Optimal Weighted Links," Proceedings of ICCS, Intl. Workshop on Comp. Geom. and Appl., pp. 649-657, 2001.

9. D. Glauser, P. Flury, Ph. Durr, H. Funakubo, C.W. Burckhardt, J. Favre, P. Schnyder and H. Fankhauser, "Configuration of a Robot Dedicated to Stereotactic Surgery," Stereotact. Func. Neurosurg., Vol. 54 and 55, pp. 468-470, 1990.

10. A. Gustafsson, B.K. Lind and A. Brahme, "A generalized pencil beam algorithm for optimization of radiation therapy," Med. Phys., Vol. 21, pp. 343-356, 1994.

11. T. Holmes and T.R. Mackie, "A comparison of three inverse treatment planning algorithms," Phys. Med. Biol., Vol. 39, pp. 91-106, 1994.

12. H. Koyama, T. Uchida, H. Funakubo, K. Takakura and H. Fankhauser, "Development of a New Microsurgical Robot for Stereotactic Neurosurgery," Stereotact. Func. Neurosurg., Vol. 54 and 55, pp. 462-467, 1990.

13. A. Schweikard, J.R. Adler and J.C. Latombe, "Motion planning in stereotaxic radiosurgery," IEEE Trans. on Robotics and Automation, Vol. 9, pp. 764-774, 1993.

14. S. Webb, Optimizing the planning of intensity-modulated radiotherapy. Phys. Med. Biol., Vol. 39, pp. 2229-2246, 1994. 